BULLETIN (New Series) OF THE

AMERICAN MATHEMATICAL SOCIETY

Volume 42, Number 2, Pages 119-135

S 0273-0979(05)01053-0

Article electronically published on January 28, 2005

\title{
WHAT IS MOTIVIC MEASURE?
}

\author{
THOMAS C. HALES
}

\begin{abstract}
This article gives an exposition of the theory of arithmetic motivic
\end{abstract} measure, as developed by J. Denef and F. Loeser.

\section{Preliminary concepts}

There is much that is odd about motivic measure if it is judged by measure theory in the sense of twentieth century analysis. It does not fit neatly with the tradition of measure in the style of Hausdorff, Haar, and Lebesgue. It is best to view motivic measure as something new and different and to recognize that when it comes to motivic measure, the term 'measure' is used loosely.

Motivic integration has been developing at a breakneck pace, ever since Kontsevich gave the first lecture on the topic in 1995. This article gives an exposition of the theory of arithmetic motivic measure, as developed by J. Denef and F. Loeser.

Motivic measure will be easier to understand once two of its peculiarities are explained. The first peculiarity is that the measure is not real-valued. Rather, it takes values in a scissor group. An introductory section on scissor groups for polygons will recall the basic facts about these groups. The second peculiarity is that rather than a boolean algebra of measurable sets, we work directly with the underlying boolean formulas that define the sets. The reasons for working directly with boolean formulas will be described in a second introductory section.

After these two introductory remarks, we will describe 'motivic counting' in Section 2. Motivic counting is to ordinary counting what motivic measure is to ordinary measure. Motivic counting will lead into motivic measure.

1.1. Scissor groups for polygons. Motivic volume is defined by a process that is similar to the scissor-group construction of the area of polygons in the plane. To draw out the similarities, let us recall the construction. It determines the area of polygons without taking limits.

Any polygon in the plane can be cut into finitely many triangles that can be reassembled into a rectangle of unit width. Figure 1illustrates three steps $(2,3$, and 4) of the general algorithm. The algorithm consists of 5 elementary transformations. (1) Triangulate the polygon. (2) Transform triangles into rectangles. (3) Fold long rectangles in half. (4) Rescale each rectangle to give it an edge of unit width.

Received by the editors June 1, 2003.

2000 Mathematics Subject Classification. Primary 14G20.

Work supported by the NSF.

This work is licensed under the Creative Commons Attribution License. To view a copy of this license, visit http://creativecommons.org/licenses/by/1.0/ or send a letter to Creative Commons, 559 Nathan Abbott Way, Stanford, California 94305, USA.

(C)2005 Thomas C. Hales 

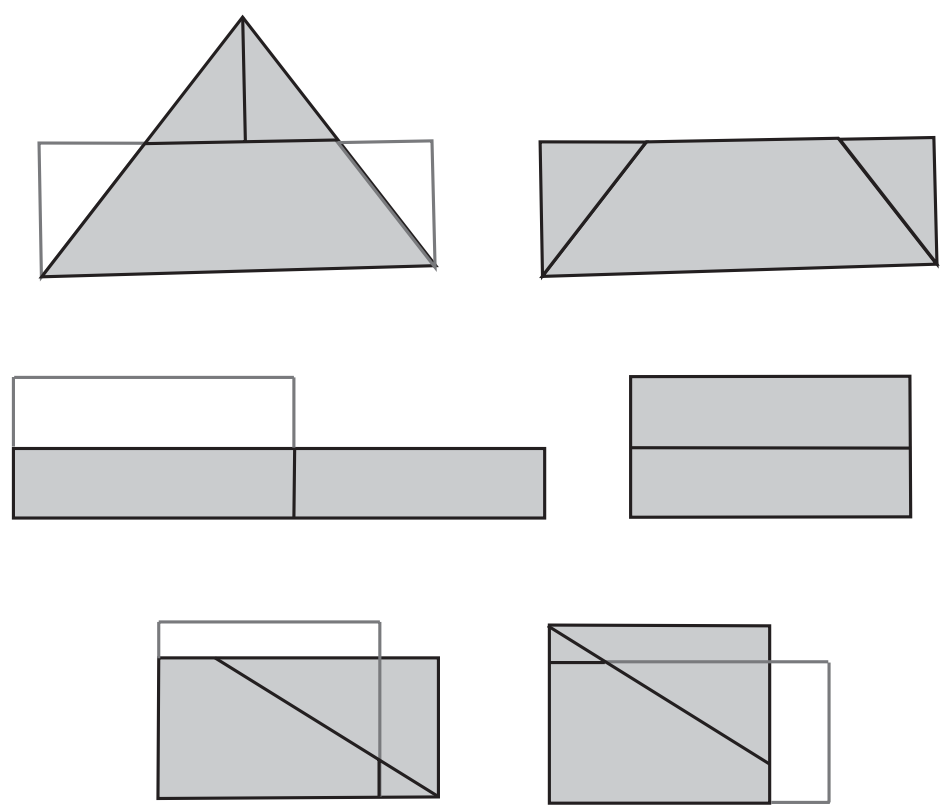

Figure 1. Triangles transform into unit width rectangles by scissor and congruence relations. Later, we will transform ring formulas into algebraic varieties by scissor and congruence relations.

(5) Stack all the unit width rectangles end to end. The length of the unit width rectangle is the area.

An abelian group encodes these cut and paste operations. Let $F$ be the free abelian group on the set of polygons in the plane.

We impose two families of relations:

Scissor relations. If $P$ is a polygon that can be cut into polygons $P_{1}$ and $P_{2}$, then

$$
[P]=\left[P_{1}\right]+\left[P_{2}\right] .
$$

Congruence relations. If $P$ and $P^{\prime}$ are congruent polygons, then

$$
[P]=\left[P^{\prime}\right] .
$$

The scissor group $\mathbb{S}_{\text {poly }}$ of polygons is defined as the free abelian group subject to these two families of relations. In some sense, this entire article is an exploration of scissor and congruence relations in diverse contexts. By and by, we will construct several closely related scissor groups $\mathbb{S}_{\text {poly }}, \mathbb{S}_{\text {count }}, \mathbb{S}_{\text {ring }}, \mathbb{S}_{\text {cover }}$, and $\mathbb{S}_{\text {mot }}$, each constructed as a free abelian group modulo scissor and congruence relations.

Theorem 1.1. The polygon scissor group $\mathbb{S}_{\text {poly }}$ of polygons is isomorphic to the additive group of real numbers $\mathbb{R}$. Under this isomorphism, the real number attached to the class $[P]$ of a polygon is its area.

Proof. A group homomorphism from $\mathbb{S}_{\text {poly }}$ to $\mathbb{R}$ sends each class $[P]$ to its area. It is onto, because there are polygons of every positive real area and negations of polygons of every negative real area. By scissor and congruence relations, every element of the scissor group is represented by the difference of two unit width 
rectangles. To be in the kernel, the two rectangles must have the same area, but then they are congruent, and their difference is the zero element of $\mathbb{S}_{\text {poly }}$. Thus, the homomorphism is also one-to-one.

The area function on the set $\{P\}$ of polygons thus factors through $\mathbb{S}_{\text {poly }}$.

$$
\begin{array}{llll}
\{P\} & \rightarrow \mathbb{S}_{\text {poly }} & \rightarrow & \mathbb{R} \\
P & \mapsto[P] & \mapsto & \text { area }(P) .
\end{array}
$$

We might ponder which of these two maps $(P \mapsto[P]$ or $[P] \mapsto$ area $(P))$ captures the greater part of the area-taking process. Motivic measure commits to a position on this issue: the first stage $(P \mapsto[P])$ is identified as the area-taking process, and the second stage $[P] \mapsto \operatorname{area}(P)$ is a specialization of the area. In this case, specialization is an isomorphism. Our approach to measure in this article is decidedly unsophisticated: taking the measure of something consists in mapping that thing into its scissor group, $P \mapsto[P]$.

1.2. The measure of a formula. Traditionally, we take the measure of a set $X=\{x \mid \phi(x)\}$ (say a subset of a locally compact space), but we do not take the measure of the formula $\phi$ defining a set. With motivic measure, we take the measure of the formula directly. Concretely, the formula

$$
\text { ' } x^{2}+y^{2}=1 \text { ' }
$$

defines the circle

$$
\left\{(x, y) \mid x^{2}+y^{2}=1\right\} .
$$

With motivic measure, we take the measure of the equation of the circle (Equation 2) rather than the measure of the circle itself (Equation 3). Attention shifts from sets to formulas.

What purpose does it serve to measure formulas rather than the underlying set? As algebraic geometers are eager to remark, each formula defines an infinite collection of sets. For instance, for each finite field $\mathbb{F}_{q}$, we can take the set of $\mathbb{F}_{q}$ points on the circle:

$$
\left\{(x, y) \in \mathbb{F}_{q}^{2} \mid x^{2}+y^{2}=1\right\}
$$

We will see that the motivic measure of the formula is a universal measure in the sense that the value it attaches to the formula does not commit us to any particular field. And yet if we are supplied with a particular field, it will be possible to recover the traditional measure of a set from the motivic measure of its defining formula. In this sense, motivic measure is to traditional measures what an algebraic variety is to its set of solutions.

\section{Counting measures And Finite fields}

Counting is the fountainhead of all measure. The measure of a finite set is its cardinality. At the risk of belaboring the point, in preparation for what is to come, let us recast ordinary counting. The scissor relation for disjoint finite sets is

$$
[X \cup Y]=[X]+[Y] \text {. }
$$

More generally, if we allow the sets to intersect, it is

$$
[X \cup Y]=[X]+[Y]-[X \cap Y] .
$$


The congruence relation asserts that

$$
[X]=\left[X^{\prime}\right]
$$

whenever there is a bijection between $X$ and $X^{\prime}$. The scissor group $\mathbb{S}_{\text {count }}$ is the quotient of the free abelian group on finite sets satisfying the scissor and congruence relations. It is isomorphic to $\mathbb{Z}$. The cardinality $\# X$ of a finite set $X$ factors through the scissor group

$$
X \mapsto[X] \mapsto \#[X] \in \mathbb{Z} .
$$

Of course, if our only purpose were to count elements in finite sets, this construction would be overkill. The first motivic measure that we present is an analogue of this approach to counting. We call it the motivic counting measure. The scissor relation will be similar to Equation 5 .

2.1. Ring formulas. Traditional measure calls for a full discussion of the class of measurable sets. Since we work with formulas rather than sets, our approach calls for a full discussion of the class of formulas to be measured.

We allow all syntactically correct formulas built from a countable collection of variables $x_{i}$, parentheses, and the symbols

$$
\forall, \quad \exists, \quad \vee, \quad \wedge, \quad \neg, \quad 0, \quad 1, \quad(+), \quad(-), \quad(*), \quad(=) .
$$

More precisely, we allow all formulas in the first-order language of rings. A formula that has been constructed from these symbols will be called a ring formula. We avail ourselves of the usual mathematical abbreviations and renamings of variables. We write 3 for $1+(1+1), x^{n}$ for $x * x * x \cdots * x$ ( $n$ times), $x y$ for $x * y, a+b+c$ for $a+(b+c)$, and so forth.

With usual abbreviations,

$$
\forall x y z \cdot x^{3}+y^{3}=z^{3},
$$

is a ring formula, because its syntax is correct. But

$$
\text { ‘) }) \forall+\forall=2 \forall((\text { ' }
$$

and

$$
\cdot \wedge \vee \wedge \vee \wedge '
$$

are not ring formulas.

2.2. The scissor group of ring formulas. We imitate the construction of the scissor groups $\mathbb{S}_{\text {poly }}$ and $\mathbb{S}_{\text {count }}$ to build the scissor group of ring formulas.

Take the free abelian group on the set of ring formulas. We impose two families of relations. The scissor relation takes the form established in Equation 5 for unions.

Scissor relations. If $\phi_{1} \vee \phi_{2}$ is a disjunction of two formulas, then

$$
\left[\phi_{1} \vee \phi_{2}\right]=\left[\phi_{1}\right]+\left[\phi_{2}\right]-\left[\phi_{1} \wedge \phi_{2}\right] .
$$

To describe the congruence relation, we must decide what it should mean for two ring formulas to be congruent. By way of analogy, in the case of polygons, two are congruent if there is a bijection between the two sets that is induced by an isometry. Our first guess at the congruence relation for ring formulas is that two ring formulas are congruent if there is a bijection between the sets of solutions for each finite field $\mathbb{F}_{q}$. (We limit ourselves to finite fields because we are attempting to 
imitate the counting measure of finite sets.) However, there are two modifications that we must make to this first guess to arrive at a workable relation.

The first modification is to use pseudo-finite fields rather than finite fields. A pseudo-finite field is an infinite perfect field such that every absolutely irreducible variety over the field has a rational point and such that there is a unique field extension of each finite degree (inside a fixed algebraic closure of the field). The defining properties of a pseudo-finite field are properties possessed by finite fields (except the part about being infinite). Moreover, logicians have found that the behavior of pseudo-finite fields is essentially no different from the generic behavior of finite fields, but they avoid the hassles that appear in positive characteristic. For those seeing pseudo-finite fields for the first time, it would not be a severe distortion of the facts to ignore the 'pseudo' and to work instead with finite fields.

The second modification is to require the bijection between the solutions to come from a ring formula that is independent of the underlying field. We are now ready to state the congruence relations.

\section{Congruence relations.}

$$
[\phi]=\left[\phi^{\prime}\right]
$$

if there exists a ring formula $\psi$ such that for every pseudo-finite field $K$ of characteristic zero, the interpretation of $\psi$ gives a bijection between the tuples in $K$ satisfying $\phi$ and the tuples in $K$ satisfying $\phi^{\prime}$.

Example 2.1. The congruence relation gives

$$
\left[{ }^{\bullet} \exists x . \quad x^{2}+b x+c=0^{\prime}\right]=\left[{ }^{\bullet} \exists X . \quad X^{2}=B^{2}-4 C^{\prime}\right] .
$$

The formula $\psi$ realizing the congruence and the bijection at the level of points is

$$
\text { ' }(b=B) \wedge(c=C) \text { '. }
$$

That is, in every pseudo-finite field of characteristic zero, a monic quadratic polynomial has a root if and only if its discriminant is a square.

Definition 2.2. The scissor group $\mathbb{S}_{\text {ring }}$ of ring formulas is defined as the free abelian group subject to the scissor and congruence relations.

\subsubsection{Counting measure.}

Definition 2.3. The counting measure of a ring formula $\phi$ is its class $[\phi]$ in the scissor group of ring formulas.

2.2.2. Fubini and Products. There is a trivial sort of Fubini theorem for finite sets: the cardinality of a Cartesian product of two sets is the product of the cardinalities of the two sets. To make sense of a Fubini theorem for ring formulas, it is necessary to introduce products to the scissor groups; that is, we need a scissor ring. This is easy to arrange. If $\phi_{1}\left(x_{1}, \ldots, x_{n}\right)$ is a formula with free variables $x_{1}, \ldots, x_{n}$ and $\phi_{2}\left(y_{1}, \ldots, y_{m}\right)$ is a formula with free variables $y_{1}, \ldots, y_{m}$, and if the free variables of $\phi_{1}$ are distinct from the free variables of $\phi_{2}$, then we declare the product to be

$$
\phi_{1}\left(x_{1}, \ldots, x_{n}\right) \wedge \phi_{2}\left(y_{1}, \ldots, y_{m}\right) .
$$

This induces a well-defined product 11 on the scissor group:

$$
\left[\phi_{1}(x)\right]\left[\phi_{2}(y)\right]=\left[\phi_{1}(x) \wedge \phi_{2}(y)\right] .
$$

\footnotetext{
${ }^{1}$ We have a moving lemma: the congruence relation in the scissor group can be used to relabel the free variables of a formula, so that free variables of the two factors are always distinct.
} 
Under this product, the scissor group becomes a ring. Equation 8 asserts that counting measure satisfies a rather trivial Fubini theorem for ring formulas - at least for ring formulas without any shared free variables.

2.2.3. The universal nature of the counting measure. The counting measure $[\phi]$ of a ring formula $\phi$ is designed to be the universal counting measure for ring formulas. For every finite field $\mathbb{F}_{q}$, there is a special counting measure on ring formulas:

$$
\phi \mapsto \#_{q}(\phi)=\#\left\{\left(x_{1}, \ldots, x_{n}\right) \in \mathbb{F}_{q}^{n} \mid \phi^{\mathbb{F}_{q}}\left(x_{1}, \ldots, x_{n}\right)\right\}
$$

It gives the number of solutions to the ring formula over a particular finite field. In contrast, the general counting measure of a ring formula takes values in a scissor ring whose construction bundles all pseudo-finite fields together.

We can be precise about the way in which the counting measure bundles the counting measures $\#_{q}(\phi)$. Each formula $\phi$ gives a function $q \mapsto \#_{q}(\phi)$, an integervalued function on the set of prime powers. Let $F$ be the ring of all integer-valued functions on the set $\left\{p^{r}\right\}$ of prime powers. Declare two functions equivalent if they take the same value at $p^{r}$ for all $r$ and for all but finitely many $p$. Write $F / \sim$ for the quotient of $F$ under this equivalence relation.

Theorem 2.4. There exists a ring homomorphism $N$ from the scissor ring $\mathbb{S}_{\text {ring }}$ to $F / \sim$ that respects counting: $\#_{*}(\phi)=N([\phi])$.

In other words, with only a finite amount of ambiguity, the counting measure specializes to counting solutions to ring formulas over finite fields. To say that $N$ is a ring homomorphism is to say that it is compatible with products and Fubini. Unlike the earlier isomorphisms for polygons $\mathbb{S}_{\text {poly }} \cong \mathbb{R}$ and finite sets $\mathbb{S}_{\text {count }} \cong \mathbb{Z}$, here we make no claim of isomorphism between the scissor group $\mathbb{S}_{\text {ring }}$ and the target $\operatorname{ring} F / \sim$.

The proof of the theorem relies on ultraproducts, a standard tool in logic.

2.3. Improving the scissor ring. The shortcoming of the scissor ring $\mathbb{S}_{\text {ring }}$ is that too much about it has been left inexplicit. In our discussion of the area of planar polygons, we found a handy set of generators (unit width rectangles). Our current aim is to find a handy set of generators of a somewhat modified scissor ring $\mathbb{S}_{\text {mot }}$. The idea is to take a ring formula, and through a process of "quantifier elimination" arrive at an equivalent ring formula that does not involve any quantifiers (that is, the symbols $\forall, \exists$ will be eliminated). A formula without quantifiers belongs less to the realm of logic than to the realm of algebraic geometry. A suggestive example of a quantifier-free formula is

$$
\left(f_{1}=0\right) \wedge\left(f_{2}=0\right) \wedge \cdots \wedge\left(f_{n}=0\right),
$$

that is, the zero set of an affine variety. In fact, we will find that the improved scissor ring is defined as a quotient of the free abelian group on the set of varieties over $\mathbb{Q}$. The details of this construction will reveal what is so motivic about motivic measure.

2.4. A scissor ring for coverings. Each ring homomorphism $f: \mathbb{S}_{\text {ring }} \rightarrow R$ defines a specialization of the counting measure

$$
\phi \mapsto[\phi] \rightarrow f[\phi] \in R .
$$

The ring $F / \sim$ is one of many possible specializations $R$.

Another specialization of $\mathbb{S}_{\text {ring }}$ comes from $n$-sheeted covers: 
Definition 2.5. We say that one formula $\phi(x)$ is an $n$-sheeted cover of another formula $\phi^{\prime}\left(x^{\prime}\right)$ if there exists a ring formula $\psi\left(x, x^{\prime}\right)$ such that for every pseudo-finite field of characteristic zero, $\psi$ gives an $n$ to 1 correspondence between the solutions $x$ of $\phi(x)$ and the solutions $x^{\prime}$ of $\phi\left(x^{\prime}\right)$.

Example 2.6. Let $\phi(x)$ be the formula ' $x \neq 0$ ' and let $\phi^{\prime}(y)$ be the formula

$$
\exists \exists .\left(z^{2}=y\right) \wedge(y \neq 0) \cdot .
$$

The formula $\psi(x, y)$ given by

$$
\text { ' } x^{2}=y '
$$

presents $\phi$ as a 2-sheeted cover of $\phi^{\prime}$.

The congruence condition for $\mathbb{S}_{\text {ring }}$ asserts that if $\phi$ is a 1 -sheeted cover of $\phi^{\prime}$, then they give the same class in $\mathbb{S}_{\text {ring. }}$. A broader congruence condition can be given as follows.

Congruence (covers). If $\phi$ is an $n$-sheeted cover of $\phi^{\prime}$ for some $n$, then

$$
[\phi]=n\left[\phi^{\prime}\right] .
$$

We may form a new scissor ring $\mathbb{S}_{\text {cover }}$ with this broader congruence condition and the old scissor relation. We have a canonical surjection $\mathbb{S}_{\text {ring }} \rightarrow \mathbb{S}_{\text {cover }}$.

2.5. The scissor group of motives. Generators. Let $\operatorname{Var}_{\mathbb{Q}}$ be the category of varieties over the field of rational numbers $\mathbb{Q}$. We take the free abelian group generated by the objects of $\operatorname{Var}_{\mathbb{Q}}$.

An example of an element of the free abelian group is $\left[\mathbb{A}^{1}\right]$, the generator attached to the affine line. This particular generator will be of special importance in the constructions that follow. We write $\mathbb{L}=\left[\mathbb{A}^{1}\right]$ for this element and for its image in various scissor groups. (The ' $\mathrm{L}$ ' is for Lefschetz, as in Lefschetz motive.)

There are two types of relations: scissor relations and congruence relations. Our scissor relation will be rather crude, but justifiably so, since the Zariski topology is a coarse topology that limits the possibilities for a scissor relation. The only cutting that will be permitted is that of partitioning a variety into a closed subvariety and its complement.

Scissor relation. If $Z$ is a closed subvariety of $X$, then

$$
[X]=[Z]+[X \backslash Z] .
$$

The congruence relation is more involved than the scissor relation. If we make a direct translation of the congruence relation for the scissor group of ring formulas, we might guess that the congruence condition between two varieties $X$ and $Y$ should be the existence of a correspondence $\Psi$ between $X$ and $Y$ that induces a bijection between $X(K)$ and $Y(K)$ for every pseudo-finite field of characteristic zero. This first guess is suggestive: the congruence relation should involve an algebraic correspondence. This suggestion lands us deep in the territory of motives. Here is the precise definition of the congruence relation.

\section{Congruence relation.}

$$
[X]=[Y]
$$


whenever $X$ and $Y$ are nonsingular projective varieties that give the same virtual Chow motive. We will uncoil this definition a bit below. All that is 'motivic' about motivic measure stems from this particular congruence relation.

Definition 2.7. The quotient of the free abelian group by the scissor and congruence relations is the motivic scissor ring $\mathbb{K}$. (The letter ' $\mathrm{K}$ ' is the standard notation for a Grothendieck group, which for our purposes is just another name for a scissor group.) The localized version $\mathbb{K}\left[\mathbb{L}^{-1}\right] \otimes \mathbb{Q}$ will be called the localized motivic scissor ring and denoted $\mathbb{S}_{\text {mot. }}$. (It will become clear in Section 3.6 .3 why it is useful to invert $\mathbb{L}$.)

It is time to uncoil the definition of this congruence relation. There is a category of Chow motives. To describe this category, we assume familiarity with the Chow groups $A^{i}(X)$ of a variety $X$. They are groups of cycles of a given codimension $i$ modulo the subgroup of cycles that are rationally equivalent to 0 . A detailed treatment of cycles, rational equivalence, and Chow groups can be found in 8 . Other good treatments of Chow motives can be found in [15] and [9].

An object in the category of Chow motives is a triple $(X, p, m)$ where $X$ is a smooth projective variety of dimension $d, p$ is an element in the Chow ring $A^{d}(X \times X)$ that is a projector $\left(p^{2}=p\right)$, and $m$ is an integer. The set of morphisms from $(X, p, m)$ to $\left(X, p^{\prime}, m^{\prime}\right)$ is defined to be the set

$$
p^{\prime} A^{d+n-m}(X \times Y) p .
$$

Varieties that are not isomorphic as varieties can very well become isomorphic when viewed as Chow motives. For example, isogenous elliptic curves are isomorphic as Chow motives.

There is a canonical morphism from the Grothendieck ring of the category $\operatorname{Var}_{\mathbb{Q}}$ to the Grothendieck ring of the category of Chow motives. We let $\mathbb{K}$ be the image of this morphism. To say that two varieties are equal as virtual Chow motives is to say that they have the same class in $\mathbb{K}$.

2.6. The motivic counting measure. The following theorem follows from a deep investigation of Chow motives and the theory of quantifier elimination for pseudofinite fields.

Theorem 2.8. There exists a unique ring homomorphism $\mathbb{S}_{\text {cover }} \rightarrow \mathbb{S}_{\text {mot }}$ that satisfies the following property (Zero Sets).

Zero Sets. If $\phi$ is a ring formula that is given by the conjunction of polynomial equations, then $[\phi]$ is sent to the affine variety defined by those polynomial equations.

There are ring homomorphisms $\mathbb{S}_{\text {count }} \rightarrow \mathbb{S}_{\text {cover }} \rightarrow \mathbb{S}_{\text {mot }}$. We use the notation $\phi \mapsto[\phi]$ for the class of $\phi$ in any of these rings, depending on the context.

Definition 2.9. The composite map $\phi \mapsto[\phi] \in \mathbb{S}_{\text {mot }}$ will be called the motivic counting measure of the formula $\phi$.

The motivic counting measure of a ring formula is thus represented by a rational linear combination of varieties over $\mathbb{Q}$. I like to think of the motivic counting measure as counting the number of solutions of the ring formula over finite fields in a way that does not depend on the finite field. Instead of giving the answer as a particular number, it gives the answer in terms of a formal combination of 
varieties having the same number of solutions over a finite field. Here is the precise statement.

Theorem 2.10. Let $\phi$ be a ring formula, and let $\sum a_{i}\left[X_{i}\right]$ be a representative of the motivic counting measure $[\phi]$ as a formal linear combination of varieties. Choose a model of each $X_{i}$ over $\mathbb{Z}$. For all $r$ and for all but finitely many primes $p$, the number of solutions of $\phi$ in $\mathbb{F}_{p^{r}}$ is equal to

$$
\sum a_{i} \# X_{i}\left(\mathbb{F}_{p^{r}}\right) .
$$

Example 2.11. As an example, let us calculate the motivic counting measure of the 'set' of nonzero cubes. The formula is given by

$$
\phi(x): \quad ' \exists y \cdot\left(y^{3}=x\right) \wedge(x \neq 0) .
$$

The scissor relation can be used to break $\phi$ into two disjoint pieces $\phi=\phi_{1} \vee \phi_{2}$ : the part $\phi_{1}$ on which -3 is a square and the part $\phi_{2}$ on which it is not. Let $\mathbb{M}$ be the class in $\mathbb{S}_{\text {mot }}$ corresponding to the zero-dimensional variety $x^{2}+3=0$. The class $\mathbb{M}$ has two solutions or no solutions according to whether -3 is a square or not. When -3 is a square, the cube roots of unity lie in the field, so that the nonzero points on the affine line give a 3 -fold cover of $\phi_{1}$ (under $y \mapsto y^{3}$ ). Thus, $\phi_{1}$ has measure

$$
\left(\frac{\mathbb{L}-1}{3}\right) \frac{\mathbb{M}}{2}
$$

On the other hand, if -3 is not a square, each non-zero element of a pseudo-finite field of characteristic zero is a cube, so that $\phi_{2}$ has measure

$$
(\mathbb{L}-1)\left(1-\frac{\mathbb{M}}{2}\right) \text {. }
$$

The sum of these two terms is the measure of $\phi$ in $\mathbb{S}_{\text {mot }}$.

\section{LOCALly COMPACT FIELDS AND HAAR MEASURES}

This section makes the transition from finite fields to locally compact fields and from counting measures to additive Haar measures.

In Section 2 we developed a universal counting measure for ring formula. It may be viewed as counting solutions to the ring formula over a finite field in a way that does not depend on the finite field.

Counting measures are a rather simple and uninteresting type of measure. In this section, we construct a universal (motivic) measure with ties to locally compact fields. This new measure may be viewed as the volume expressed in a way that does not depend on the locally compact field. To carry out the construction, we must work with a different collection of formulas (called DVR formulas) that are better adapted to locally compact fields. 'DVR' is an acronym for discrete valuation ring.

3.1. Examples of rings. To make the transition from finite fields to locally compact fields, we wish to replace ring formulas with formulas in a language that has a rich assortment of locally compact structures.

Example 3.1. Let $\mathbb{C}[[t]]$ be the ring of formal power series with complex coefficients. A typical element of this ring has the form

$$
x=\sum_{i=k}^{\infty} a_{i} t^{i}
$$


(with no constraints on the convergence of the series). Pick the initial index $k$ so that $a_{k} \neq 0$ (if $x \neq 0$ ).

The valuation of $x$ is defined to be the integer $k$ :

$$
\operatorname{val}(x)=k \text {. }
$$

The angular component of $x$ is defined to be the complex number $a_{k}$.

$$
\operatorname{ac}(x)=a_{k} \in \mathbb{C}^{\times} .
$$

(In the special case $x=0$, we set $\operatorname{val}(0)=\infty$ and $\operatorname{ac}(0)=0$.)

The name angular component is not meant to suggest any precise connection to angles. The name is based on a loose analogy with the polar coordinate representation of a complex number: just as the angular component $\theta$ of a nonzero complex number $r e^{i \theta}$ distinguishes among complex numbers of the same magnitude (or valuation) $r$, so the angular component of a formal power series helps to distinguish among formal power series of a given valuation $k$.

There are many other rings with similar functions, ac and val. For example, we can change the coefficient ring of the formal power series from $\mathbb{C}$ to any other field $k$ to obtain $k[[t]]$. Or we can take the field of fractions of $k[[t]]$, which is the field of formal Laurent series with coefficients in $k$ :

$$
k((t))=\left\{\sum_{-N}^{\infty} a_{i} t^{i} \mid a_{i} \in K\right\} .
$$

For each prime $p$, there are valuation and angular component functions defined on the field of rational numbers. If $x$ is a nonzero rational number, pick integers $a, b, c, N$ so that

$$
x=a p^{N}+\frac{b p^{N+1}}{c},
$$

where $c$ is not divisible by $p$, and $a \in\{1, \ldots, p-1\}$. The integers $a$ and $N$ are uniquely determined by this condition. Define the valuation of $x$ to be $\operatorname{val}_{p}(x)=$ $N \in \mathbb{Z}$ and the angular component of $x$ to be the image of $a$ modulo $p$ in $\mathbb{F}_{p}$.

Example 3.2. If $p=2$ and $x=17 / 8$, then

$$
17 / 8=1.2^{-3}+2, \quad \operatorname{val}_{2}(17 / 8)=-3, \quad \text { ac }(17 / 8)=1 \in \mathbb{F}_{2} .
$$

Other examples can be obtained from this one by completion. For each $p$,

$$
d(x, y)=(1 / 2)^{\operatorname{val}_{p}(x-y)}
$$

is a metric on the set of rational numbers. The completion is a locally compact field, called the field of $p$-adic numbers $\mathbb{Q}_{p}$. The valuation val $p$ and angular component function ac extend to the completion.

3.2. The DVR language. We have seen by example that there are many rings with functions val and ac. In each case, there are three separate rings that come into play: the domain of the functions val and ac, the range of the function val (which we augment with a special symbol $\{\infty\}$ for the valuation of 0 ), and the range of the function ac. We call these rings the valued ring, the value group, and the residue field, respectively.

We formalize this relationship as a language in first-order logic with function symbols val and ac. We allow ourselves to build syntactically well-formed expressions with variables, parentheses, quantifiers, the function symbols val and ac, the 
usual ring operations $(0,1,(+),(-),(*),(=))$ on the valued ring and residue field, and the usual group operations and inequalities on the value group $(0,(+),(\leq))$. These formulas will use variables of three different types: $x_{i}$ for the value ring, $m_{i}$ for the value group, and $\xi_{i}$ for the residue field. Quantifiers $\forall, \exists$ can be used to bind all three sorts of variables.

The construction of first-order languages is commonplace in logic, but even without any background in logic, it is not hard to guess whether a formula is syntactically correct. We allow standard mathematical abbreviations similar to those introduced above for ring formulas.

$$
\bullet \forall y \cdot\left(\exists x \cdot x^{2}=y\right) \Longrightarrow(\exists m \cdot 2 m=\operatorname{val}(y)),
$$

is syntactically correct. But

$$
' \forall f . \forall x . \forall y \cdot f(y, \operatorname{ac}(y)) '
$$

is not well-formed, because quantifiers are not allowed over higher-order relations $f$ in a first-order language. Also,

$$
' \forall x \xi .(0 \leq x) \vee(\operatorname{ac}(x)=\xi) '
$$

is not well-formed, because of a type error; the variable symbol $x$ appears once as an integer $0 \leq x$ and again as a variable in the valued field ac $(x)$.

A syntactically correct formula is called a DVR formula. The aim of motivic measure is to compute the "volume" of a DVR formula in a universal way, that is, in a way that does not depend on the underlying locally compact field.

3.3. Assumptions on the ring. The various examples that we have mentioned are all structures for the DVR language: rings of formal power series $k[[t]]$ and fields of formal Laurent series $k((t))$. For each prime $p,\left(\mathbb{Q}, \mathrm{ac}_{\mathrm{c}}, \mathrm{val}_{p}\right)$ is a structure for the language, as well as its completion $\left(\mathbb{Q}_{p}, \mathrm{ac}, \mathrm{val}_{p}\right)$.

We will temporarily restrict the set of examples to structures $(K, k$, ac, val) that satisfy the following conditions.

- $K$ is a valued field of characteristic zero, with valuation function val : $K \rightarrow$ $\mathbb{Z} \cup\{\infty\}$ and angular component functions ac $: K \rightarrow k$.

- The residue field $k$ has characteristic zero.

- $K$ is henselian. (We review the definition below.)

Examples that satisfy these conditions include the fields $k((t))$, where $k$ has characteristic zero. The analogy that will guide us is that these fields stand in the same relation to locally compact DVR fields as pseudo-finite fields do to finite fields.

3.4. Henselian field. There is only one plausible definition for a henselian field: A field is henselian if the field satisfies Hensel's lemma.

Hensel's lemma gives checkable conditions on a polynomial that insure that it has a root in a given neighborhood. Hensel's lemma occupies the same ground in the realm of DVR rings that the intermediate value theorem occupies in the realm of real numbers. (The intermediate value theorem also gives checkable conditions on a polynomial that insure that it has a real root in a given neighborhood.)

Our experience with motivic counting measures has alerted us to the importance of quantifier elimination, that is, the process of replacing a formula with quantifiers $\forall, \exists$ with an equivalent formula that does not contain quantifiers. The simplest case of quantifier elimination is the determination of when there exists a root of a 
polynomial. Without a criterion for the existence of roots to polynomials, quantifier elimination would be impossible. For the pseudo-finite fields, this is handled through the defining property of pseudo-finite fields that "every absolutely irreducible variety has a root". For real fields, quantifier elimination is based on the intermediate value theorem. For henselian fields, quantifier elimination is based on Hensel's lemma.

Lemma 3.3. (Hensel's lemma) For every monic polynomial $f \in K[x]$ whose coefficients have non-negative valuation, and for every $x$ such that

$$
\operatorname{val}(f(x))>0
$$

and

$$
\operatorname{val}\left(f^{\prime}(x)\right)=0,
$$

there exists $y \in K$ such that $f(y)=0$ and $\operatorname{val}(y-x)>0$.

This is stated as a lemma, but we view it as a condition on the field $K$ and its valuation. It can be proved that the fields $k((t))$ and $\mathbb{Q}_{p}$ are henselian by showing that under the hypotheses of Hensel's lemma, Newton's approximations to the roots

$$
\begin{array}{ll}
x_{0} & =x \\
x_{n+1} & =x_{n}-f\left(x_{n}\right) / f^{\prime}\left(x_{n}\right)
\end{array}
$$

converge to a root.

\subsection{Quantifier elimination.}

Theorem 3.4. (14) Let $K$ be a field satisfying the other conditions enumerated in 3.3 with residue field $k$. Let $\phi$ be a DVR formula. Then there is another formula $\phi^{\prime}$ without quantifiers of the valued field sort such that

$$
\forall(x, \xi, m) \in K^{n} \times k^{m} \times(\mathbb{Z} \cup\{\infty\})^{r} . \quad \phi^{K}(x, \xi, m)=\phi^{\prime}{ }^{K}(x, \xi, m) .
$$

Moreover, the formula $\phi^{\prime}$ can be chosen to be independent of the structure $K$.

3.6. Outer measure of a DVR formula. As a first step toward constructing the measure of a DVR formula, we will define an outer measure of a formula. To motivate this construction, it might be helpful first to describe an analogous construction in Euclidean space.

3.6.1. An outer measure in Euclidean space. Fix a positive integer $m$. Tile Euclidean space with cubes of width $1 / 2^{m}$ whose vertices are centered at points $a$ with coordinates $a_{i} \in \mathbb{Z} / 2^{m}$.

According to the Calculus 101 approach to volume, we can approximate the volume of a set by counting the number of cubes that it meets. Let $A$ be a bounded set in $\mathbb{R}^{n}$. Let $C_{m}(A)$ be the set of cubes in this tiling that meet $A$. In our naive approach to measure, let us define the outer measure of $A$ at level $m$ in dimension $n$ to be

$$
\frac{\# C_{m}(A)}{2^{m n}}
$$

that is, the number of cubes divided by the scaling factor $2^{m n}$. (If doing so did not involve logical circularity, we would identify $1 / 2^{m n}$ with the volume of the cube and the entire expression as the volume of the set $C_{m}(A)$ of cubes.)

The outer motivic measure of a DVR formula will be formed in an entirely analogous way. Of course, we will need to decide what to use for cubes, how to 




Figure 2. Volumes of DVR formulas can be approximated in Calculus 101 fashion by counting centers of cubes that meet a given formula, scaled according to the size of the cubes.

count the number of cubes that "meet" a given formula, and what scaling factor to use. Once we make these decisions, the formula for outer measure will take precisely the same form as Equation 10,

In the planar case, we gave a construction of the area of polygons as taking values in a scissor group $\mathbb{S}_{\text {poly }}$. The outer approximation of any bounded planar set $A$ by squares gives a value in the scissor group of polygons. Here too, if our outer approximation to a DVR formula is with a ring formula, then the value of the outer measure of the DVR formula will be in a scissor ring $\mathbb{S}_{\text {mot }}$.

Given all our preliminaries, it almost goes without saying that the number of cubes appearing in the numerator of Equation 10 will be replaced with the motivic counting measure of a ring formula.

3.6.2. Cubes. What is a cube? Well, it is a product of equal width intervals. In DVR formulas, a cube centered at $a$ of "width" $m$ is again a product of intervals:

$$
\left\{\left(x_{1}, \ldots, x_{n}\right) \in K^{n} \mid \operatorname{val}\left(x_{i}-a_{i}\right) \geq m, \quad \text { for } i=1, \ldots, n\right\} .
$$

If $K=k[[t]]$, then the interval around a formal power series $a$ is the set of all formal power series with the same leading terms. Shaking (wagging) the tails of the power series fills out the interval. In other words, we can make precise the idea of covering a DVR formula with cubes by replacing each solution to the DVR formula with a bigger set where the tails of the solutions are allowed to vary.

Let us make this precise. We have truncation map

$$
\begin{aligned}
& k[[t]] \rightarrow k[[t]] /\left(t^{m}\right) \simeq k^{m} \\
& \sum_{0}^{\infty} a_{i} t^{i} \mapsto \sum_{0}^{m-1} a_{i} t^{i} \mapsto\left(a_{0}, \ldots, a_{m-1}\right) .
\end{aligned}
$$

In the opposite direction, given $b \in k^{m}$, there is a polynomial with those coefficients

$$
p(b, t)=\sum_{0}^{m-1} b_{i} t^{i} \in k[[t]]
$$

Definition 3.5. Let $\phi$ be a DVR formula with free variables $\left(x_{1}, \ldots, x_{n}\right)$ and no free variables of other sorts. An outer ring formula $\phi_{m}$ approximation to $\phi$ (at level $m$ ) is a ring formula in $n m$ free variables $u_{i j}$ such that over every field $k$ :

$$
\begin{aligned}
& \left\{u \in k^{n m} \mid \phi_{m}(u)\right\}= \\
& \quad\left\{u \in k^{n m} \mid \exists a_{1}, \ldots, a_{n} . \phi\left(a_{1}, \ldots, a_{n}\right) \wedge \operatorname{val}\left(a_{i}-p\left(u_{i j}, t\right)\right) \geq m\right\} .
\end{aligned}
$$


This set is the set of centers of cubes that contain a solution to $\phi$.

Theorem 3.6. Outer ring formula approximations exist for every $D V R$ formula $\phi$ at every level $m$.

The proof of this theorem uses quantifier elimination results to eliminate the quantifiers that bind variables ranging over the valued field. It uses results of Presburger on quantifier elimination to eliminate the quantifiers that range over the additive group of integers. The quantifiers that bind variables in the residue field remain as quantifiers in the ring formula $\phi_{m}$.

3.6.3. Scaling factors. How is the scaling factor chosen in Equation 10 for Euclidean outer measures? The scaling factor $1 / 2^{n m}$ is the unique constant that has the property that if the set $A$ is itself a union of properly aligned cubes (of width $m^{\prime}$ ), then the outer measure of $A$ is independent of $m$ for all $m \geq m^{\prime}$.

To find the scaling factor for DVR formulas, we work a simple example in which the DVR formula is itself a union of cubes of width $m^{\prime}$ (that is, its set of solutions is stable under perturbation of the power series tails).

Example 3.7. Let $\phi\left(x_{1}, \ldots, x_{n}\right)=\mathbb{T}$, a formula that is true for all values of the free variables $x_{i}$. In this case the outer ring formula approximation is exact. Substitute polynomials $p\left(u_{i}, t\right)$ for each $x_{i}$ and expand in terms of $m n$ distinct free variables $u_{i j}$ to get

$$
\phi_{m}\left(u_{i j}\right)=\mathbb{T}
$$

for all input values $u_{i j}$. The number of solutions of $\phi_{m}$ over a finite field $\mathbb{F}_{q}$ is $q^{n m}$. If we take the motivic counting measure of $\phi_{m}$, we find that the variety that counts the points of $\phi_{m}$ over any finite field is the affine space of dimension $\mathrm{nm}$ :

$$
\# \mathbb{A}^{n m}\left(\mathbb{F}_{q}\right)=q^{n m}
$$

The class of $\phi_{m}$ in $\mathbb{K}\left[\mathbb{L}^{-1}\right] \otimes \mathbb{Q}$ is

$$
\left[\mathbb{A}^{n m}\right]=\left[\mathbb{A}^{1}\right]^{n m}=\mathbb{L}^{n m} .
$$

From this one example, we see that the scaling factor for DVR formulas must be $1 / \mathbb{L}^{n m}$.

Definition 3.8. Let $\phi$ be a DVR formula. Let the outer measure of $\phi$ at level $m$ be given by

$$
\frac{\left[\phi_{m}\right]}{\mathbb{L}^{n m}} \in \mathbb{K}\left[\mathbb{L}^{-1}\right] \otimes \mathbb{Q}=\mathbb{S}_{\mathrm{mot}}
$$

This formula is analogous to Formula 10 for the Euclidean outer measure at level $m$. The numerator counts the number of centers of cubes that contain a solution to the DVR formula.

Definition 3.9. Let the motivic measure (or motivic volume) of $\phi$ be given by

$$
\lim _{m \rightarrow \infty}\left[\phi_{m}\right] \mathbb{L}^{-n m}
$$

whenever that limit exists. (The limit must be taken in a completion of $\mathbb{S}_{\text {mot }}$.) 
3.7. The universal nature of motivic measure. Just as the motivic counting measure counts solutions to ring formulas over finite fields in a field independent way, so the motivic measure takes the volume of a DVR formula over locally compact fields in a field independent way 2

There is a good theory of measure on locally compact fields. This is the Haar measure, which is translation invariant. Given a DVR formula $\phi$ and a locally compact structure $K$ with ring of integers $O_{K}$, we can take the volume of the set of solutions to the DVR formula

$$
\operatorname{vol}\left(\left\{x \in O_{K}^{n} \mid \phi^{K}(x)\right\}, d x\right) .
$$

The measure $d x$ can be given a canonical normalization by requiring that it assigns volume 1 to the full set $O_{K}^{n}$.

We are now ready to state the main result on motivic measure. Like all the other principal results in this article, the result is due to J. Denef and F. Loeser.

Theorem 3.10. The motivic volume of $\phi$ is universal in the following sense. Let $\sum a_{i}\left[X_{i}\right] \mathbb{L}^{-N_{i}}$ be any representative of the motivic volume of $\phi$ as a convergent formal sum of varieties over $\mathbb{Q}$. Pick models for the varieties over $\mathbb{Z}$. After discarding finitely many primes, for any locally compact structure of the DVR language, the $K$-volume of the formula is given by a convergent sum (in $\mathbb{R}$ )

$$
\sum a_{i} \# X\left(\mathbb{F}_{q}\right) q^{-N_{i}}
$$

where $\mathbb{F}_{q}$ is the residue field of $K$.

This wonderful result states that the Haar measures on all locally compact fields have a deep underlying unity. The volumes of sets can be expressed geometrically in a way that is independent of the underlying field.

Moreover, there are effective procedures to calculate the varieties $X_{i}$ and the coefficients $a_{i}, N_{i}$ that represent the outer motivic volume at level $m$. If the outer ring formula approximations $\phi_{m}$ converge at some finite level $m$ to the DVR formula $\phi$, then we obtain effective procedures to calculate the motivic volume of the formula.

\section{Applications and COnclusions}

What good is motivic measure? Here are a few examples.

4.1. Invariants of ring formulas. The group $\mathbb{S}_{\text {mot }}$ is generated by varieties $\operatorname{Var}_{\mathbb{Q}}$. Many geometrical invariants of varieties (such as Euler characteristics and Hodge polynomials) can be reformulated as invariants of the ring $\mathbb{S}_{\text {mot }}$. This gives a novel way to attach invariants to every ring formula $\phi$ : take a geometric invariant of $[\phi] \in \mathbb{S}_{\text {mot }}$. In particular, ring formulas have Euler characteristics and Hodge polynomials! For example, the formula for the nonzero squares in a field

$$
' \exists y .\left(y^{2}=x\right) \wedge(x \neq 0)^{\prime}
$$

has Euler characteristic zero.

\footnotetext{
${ }^{2}$ It is impossible for the structure $K$ both to be locally compact and to have a residue field $k$ of characteristic zero, as required by Condition 3.3 The residue field of a locally compact field is always finite. In these final paragraphs, we allow the residue field to have positive characteristic.
} 
4.2. Geometry of varieties. There is a motivic change-of-variables formula that is similar to the standard change of variables formula in calculus. Using this formula, it is sometimes possible to show that two birationally equivalent varieties have the same motivic volume. This has deep implications for the geometry of the two varieties. In particular, the motivic volume determines the Hodge polynomial of the varieties.

This approach was followed by Kontsevich, who used a change-of-variables calculation to show that birationally equivalent projective Calabi-Yau manifolds have the same Hodge numbers [10]. Applications to orbifolds appear in [13].

4.3. Computation of $p$-adic integrals. Many integrals over $p$-adic fields are notoriously difficult to calculate. Motivic measure exposes the underlying similarities between volumes on different $p$-adic fields. It gives a decision procedure to calculate $p$-adic integrals (at least when the data defining the integral can be expressed as DVR formulas that can be reproduced at some finite level $m$ ). In particular, this means that a computer can be programmed to compute a large class of $p$-adic integrals.

4.4. Generating functions. Motivic counting gives a way of counting that is independent of the finite field. Let

$$
Z_{p}(t)=\sum_{i=0}^{\infty} a_{i}^{(p)} t^{i}
$$

be a generating function, where the constants $a_{i}^{(p)}$ are obtained by counting solutions to a formula in some $p$-dependent way. (Each generating function depends on a single prime $p$.) Motivic measure can often give a way of forming a $p$-independent series

$$
Z_{\mathrm{mot}}(t)=\sum_{i=0}^{\infty}\left[a_{i}\right] t^{i}
$$

taking values in $\mathbb{S}_{*}[[t]]$ and specializing for almost all $p$ to the $p$-dependent series $Z_{p}(t)$. The motivic series collects the behavior of the various series $Z_{p}(t)$ into a single series.

Denef and Loeser have studied motivic versions of Hasse-Weil series, Igusa series, and Serre series. They have used the general motivic series to prove that various properties of these series are independent of the prime $p$. See [7].

4.5. Concluding remarks. This article is an exposition of a particular version of motivic integration, called arithmetic motivic integration. Proofs of results stated in this article can be found in [6] and [5]. Motivic integration has been developing at a breakneck pace, ever since Kontsevich gave the first lecture on the topic in 1995. The version of motivic integration developed in the late nineties goes by the name of geometric motivic integration. Geometric motivic integration is a coarser theory, but is sufficient for many applications. Good introductions are [2] and [12]. Some articles on geometric motivic integration include [4] and [3]. Another version of motivic integration has been developed by J. Sebag for formal schemes [16]. See also [11]. Cluckers and Loeser are in the final stages of preparation of an ultimate version of motivic integration that subsumes both geometric and arithmetic motivic integration [1]. 
We began this article by stating that motivic measure does not fit neatly into the tradition of Hausdorff, Haar, and Lebesgue. However, a major result states that the motivic measure specializes to the additive Haar measure on locally compact fields (Theorem 3.10). Thus, the motivic measure is perhaps not so peculiar after all. In fact, in many respects it is strikingly similar to the additive Haar measure on locally compact fields. It has been my experience when I calculate motivic volumes to lose track - mid-calculation - of which measure is being used.

\section{REFERENCES}

[1] R. Cluckers and F. Loeser, Fonctions constructibles et intégration motivique I, II, C. R. Math. Acad. Sci. Paris 339 (2004), no. 6, 411-416. MR2092754

[2] A. Craw, An introduction to motivic integration, 1999.

[3] J. Denef and F. Loeser, Motivic Igusa functions, Journal of Algebraic Geometry 7, 505-537 (1998). MR1618144 (99j:14021)

[4] J. Denef and F. Loeser, Germs of arcs on singular algebraic varieties and motivic integration, Inventiones Mathematicae 135, 201-232 (1999). MF.1664700 (99k:14002)

[5] J. Denef and F. Loeser, Definable sets, motives and $p$-adic integrals, JAMS 14, No. 2, 429-469, 2000. MR $1815218(2002 \mathrm{k}: 14033)$

[6] J. Denef and F. Loeser, Motivic integration and the Grothendieck group of pseudo-finite fields, Proc. ICM, Vol. II (Beijing, 2002), 13-23, Higher Education Press, 2002. MR 1957016 (2004f:14040)

[7] J. Denef and F. Loeser, On some rational generating series occurring in arithmetic geometry, to appear.

[8] W. Fulton, Intersection Theory, second edition, Springer, 1998. MR/1644323 (99d:14003)

[9] G. van der Geer and B. Moonen, Abelian Varieties, preliminary version of Chapter XI. The Fourier transform and the Chow ring, July 2003, http://turing.wins .uva.nl/f̃moonen/boek/BookAV.html .

[10] M. Kontsevich, lecture at Orsay, December 1995.

[11] F. Loeser and J. Sebag, Motivic integration on smooth rigid varieties and invariants of degenerations, Duke Mathematical Journal 119, 315-344 (2003). MR/1997948 (2004g:14026)

[12] E. Looijenga, Motivic measures. Séminaire Bourbaki, Vol. 1999/2000. Astérisque No. 276 (2002), 267-297. MR1886763 (2003k:14010)

[13] E. Lupercio and M. Poddar, The global McKay-Ruan correspondence via motivic integration, Bull. London Math. Soc. 36 (2004), no. 4, 509-515. MR2069013

[14] J. Pas, Uniform $p$-adic cell decomposition and local zeta functions, J. reine angew. Math. 399 (1989), 137-172. MR1004136 (91g:11142)

[15] A. Scholl, Classical motives, in Motives (U. Jannsen, S. Kleiman, J-P. Serre, Eds.), Proc. Symp. Pure Math., Vol. 55, Part 1 (1994), 163-187, Amer. Math. Soc. MR1265529 (95b:11060)

[16] J. Sebag, Intégration motivique sur les schémas formels, Bull. Soc. Math. France 132 (2004), no. 1, 1-54. MR2075915

Department of Mathematics, University of Pittsburgh, Pittsburgh, Pennsylvania 15260 